Research Article

\title{
Viscoelastoplastic Displacement Solution for Deep Buried Circular Tunnel Based on a Fractional Derivative Creep Model
}

\author{
Yi-Hang Gao, ${ }^{1,2}$ Zhou Zhou, ${ }^{1,2}$ Hang Zhang, ${ }^{1,2}$ Shuang Jin $\left(D,{ }^{3}\right.$ Wen Yang, ${ }^{3}$ \\ and Qing-Hua Meng ${ }^{4}$ \\ ${ }^{1}$ State Key Laboratory of Geohazard Prevention and Geoenvironment Protection (Chengdu University of Technology), \\ Chengdu 610059, China \\ ${ }^{2}$ College of Environment Geology and Civil Engineering, Chengdu University of Technology, Chengdu 610059, China \\ ${ }^{3}$ Fifth Geological Brigade, Hebei Bureau of Geology and Mineral Resources, Tangshan 063000, China \\ ${ }^{4}$ Tianjin Center, China Geological Survey, Tianjin 300000, China \\ Correspondence should be addressed to Shuang Jin; jinshuang19870202@gmail.com
}

Received 22 July 2021; Revised 15 August 2021; Accepted 16 August 2021; Published 16 September 2021

Academic Editor: Guowen Xu

Copyright ( 2021 Yi-Hang Gao et al. This is an open access article distributed under the Creative Commons Attribution License, which permits unrestricted use, distribution, and reproduction in any medium, provided the original work is properly cited.

\begin{abstract}
Time-dependent deformation of surrounding rock is a common phenomenon for tunnels situated in soft rock stratum or hard rock stratum with high geo-stress. To describe this phenomenon, a creep model combining the Abel dashpot and a non-Newton viscous element was adopted, and the analytical solution about the viscoelastoplastic deformation for circular tunnel was obtained based on this creep model. Then, the auxiliary tunnel of Jinping II hydropower station was taken as an example to reveal the influence of creep parameters on the creep deformation. The research shows that (1) the creep model can well describe the whole creep stage of rocks, that is, the decay, constant, and accelerated creep stages, (2) the creep deformation has a positive relation with the value of fractional order of Abel dashpot and the order of the non-Newton viscous element, and (3) the creep curves between test results and analytical solutions are well consistent with each other, which demonstrate the validity of the analytical solution.
\end{abstract}

\section{Introduction}

The rheological deformation of surrounding rock is a common issue encountered in the excavation of soft rock tunnels under the conditions of high in situ stress. When the rheological deformation exceeds a certain level, the surrounding rock will be squeezed, which causes the failure of supporting structures and adversely affects the safety of tunnel constructions and long-term operations [1]. Therefore, the analysis regarding the rheological effect of surrounding rock and its influence on the viscoelastic-plastic process of tunnel deformation has important theoretical and practical significance.

Researchers have conducted a lot of analytical studies on the creep features of tunnels. Hong et al. [2] obtained the rheological deformation solution of an axisymmetric circular roadway tunnel based on the Nishihara model. Tang et al. [3] derived the time-dependent displacement solution of deep-buried tunnels considering the effects of strain strengthening and dilation of surrounding rock using a viscoelastic-plastic creep model which is comprised of Burgers body and Drucker-Prager yield criterion. Zhang et al. [4] adopted the improved Nishihara model to derive the viscoelastic-plastic creep displacement of seepage tunnels under the influence of the expansion strain of the surrounding rock. Gu and Yan [5] studied the creep behavior of surrounding rock under the action of seepage with the improved Burgers model. Deleruyelle et al. [6] used the Norton-Hoff criterion to describe the creep effect of surrounding rock and derived an analytical solution for tunnel displacement considering the postpeak effect of surrounding rock.

In former research, the creep behavior of surrounding rock is generally described by viscoelastic constitutive models. However, none of these models can well reflect the accelerated deformation stage of rocks $[7,8]$. Few 
researchers have done pioneer work in deriving the closedform solution of tunnel displacement using viscoelastoplastic models $[9,10]$. Therefore, in this article, a creep model, which consists of an elastic element, a non-Newtonian viscous element, an Abel element based on the fractional calculus theory, and a plastic element, is adopted to simulate the whole creep deformation stage of rocks. Then, the viscoelastic displacement solution of the deepburied tunnel is derived based on this creep model. Finally, taking the auxiliary tunnel of Jinping II Hydropower Station as an example, the influence of rheological parameters on the tunnel deformation is discussed.

\section{Creep Constitutive Model}

2.1. Abel Element Based on Fractional Theory. Fractional calculus operation refers to that the order of integration or differentiation of a function is any real or complex number [11]. Among all relevant definitions, the Riemann-Liouville definition is most suitable for studying the viscosity characteristics of materials. The integral of function $f(t)$ with an order of $\gamma$ is

$$
\frac{\mathrm{d}^{-\gamma}[f(t)]}{\mathrm{d} t^{-\gamma}}={ }_{t_{0}} D_{t}^{-\gamma} f(t)=\frac{1}{\Gamma(\gamma)} \int_{t_{0}}^{t}(t-\tau)^{\gamma-1} f(\tau) \mathrm{d} \tau .
$$

The corresponding differentiation is

$$
\frac{\mathrm{d}^{\gamma}[f(t)]}{\mathrm{d} t^{\gamma}}={ }_{t_{0}} D_{t}^{\gamma} f(t)=\frac{\mathrm{d}^{n}\left[{ }_{t_{0}} D_{t}^{-(n-\gamma)} f(t)\right]}{\mathrm{d} t^{n}},
$$

where $\gamma>0$ and $n-1<\gamma \leq n$ ( $n$ is the smallest positive integer which is larger than $\gamma$ ) and $\Gamma(\gamma)$ is the Gamma function with expression of $\int_{0}^{\infty} e^{-t} t^{\gamma-1} \mathrm{~d} t$.

The Abel dashpot based on the theory of fractional calculus can well describe the viscous characteristics of the viscous body between the ideal elastic body and the ideal fluid, and its expression is

$$
\sigma(t)=\eta \frac{\mathrm{d}^{\gamma} \mathcal{E}(t)}{\mathrm{d} t^{\gamma}}
$$

When $\sigma(t)$ is a constant, its creep equation is

$$
\varepsilon(t)=\frac{\sigma}{\eta^{\gamma}} \frac{t^{\gamma}}{\Gamma(1+\gamma)}(0 \leq \gamma \leq 1)
$$

2.2. Non-Newtonian Viscous Element. The NVPB element [12] is put forward to describe the accelerated deformation process of rock after it enters the plastic stage, and its creep equation is

$$
\varepsilon(t)=\frac{H\left(\sigma_{0}-\sigma_{s}\right)}{\eta} \frac{t^{n}}{t_{0}^{n-1}}=\frac{H\left(\sigma_{0}-\sigma_{s}\right)}{\eta} t^{n},
$$

where $t_{0}$ is reference time with a value of $1, n$ is the creep index, $\sigma_{s}$ is the long-term strength of rock, and $H(\sigma)$ is unit step function:

$$
H\left(\sigma_{0}-\sigma_{s}\right)= \begin{cases}0, & \sigma_{0} \leq \sigma_{s} \\ \sigma_{0}-\sigma_{s}, & \sigma_{0}>\sigma_{s}\end{cases}
$$

2.3. The Creep Model. A creep model (Figure 1) combining the Abel dashpot and a non-Newton viscous element was adopted to simulate the whole creep deformation stage of rocks.

The stress-strain relation of each element under the condition of viscoelastic state is [9]

$$
\left\{\begin{aligned}
\sigma_{1} & =E_{1} \varepsilon_{1}, \\
\sigma_{2} & =\eta_{2}^{\gamma} \frac{d^{\gamma} \varepsilon_{2}}{d t^{\gamma}}, \sigma_{0}<\sigma_{s}, \\
\sigma & =\sigma_{1}=\sigma_{2}, \\
\varepsilon & =\varepsilon_{1}+\varepsilon_{2} .
\end{aligned}\right.
$$

The stress-strain relation of each element under the condition of viscoplastic (the plastic behavior of rocks is described by the Hoke-Brown criterion) state is

$$
\left\{\begin{array}{l}
\sigma_{1}=E_{1} \varepsilon_{1}, \\
\sigma_{2}=\eta_{2}^{\gamma} \frac{\mathrm{d}^{\gamma} \varepsilon_{2}}{\mathrm{~d} t^{\gamma}}, \\
\sigma_{3}=\sigma_{s}+\eta_{2} \frac{\varepsilon_{3}}{\left(n t^{n-1}\right)}, \sigma_{0} \geq \sigma_{s}, \\
\sigma=\sigma_{1}=\sigma_{2}=\sigma_{3}, \\
\varepsilon=\varepsilon_{1}+\varepsilon_{2}+\varepsilon_{3},
\end{array}\right.
$$

where $\sigma$ and $\varepsilon$ are the total stress and strain, respectively, $\sigma_{1}$, $\sigma_{2}$, and $\sigma_{3}$ are the stress of element 1,2 , and 3, respectively, $\varepsilon_{1}, \varepsilon_{2}$, and $\varepsilon_{3}$ are the strain of element 1,2 , and 3, respectively, and $E_{1}$ the elastic modulus of element 1 .

(1) The stress-strain relationship of the elastic element:

$$
\varepsilon_{1}=\frac{\sigma}{E_{1}} \text {. }
$$

(2) The stress-strain relationship of the Abel dashpot [9]:

$$
\varepsilon_{2}=\frac{\sigma}{\eta^{\gamma}} \frac{t^{\gamma}}{\Gamma(1+\gamma)} \text {. }
$$

(3) The stress-strain relationship of the NVPB element [12]:

$$
\varepsilon_{3}=\frac{\sigma-\sigma_{s}}{\eta_{3}} t^{n}
$$

Thus, the constitutive model of the creep model is 


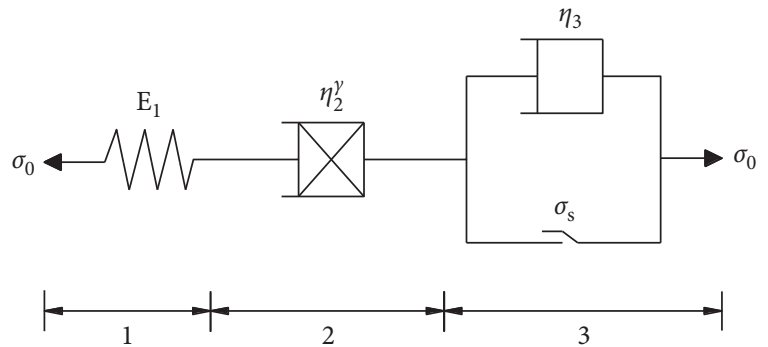

Figure 1: Creep constitutive model.

$$
\begin{aligned}
& \varepsilon(t)=\frac{\sigma}{E}+\frac{\sigma}{\eta^{\gamma}} \frac{t^{\gamma}}{\Gamma(1+\gamma)}\left(\sigma<\sigma_{s}\right), \\
& \varepsilon(t)=\frac{\sigma}{E}+\frac{\sigma}{\eta^{\gamma}} \frac{t^{\gamma}}{\Gamma(1+\gamma)}+\frac{\sigma-\sigma_{s}}{\eta_{3}} t^{n}\left(\sigma \geq \sigma_{s}\right) .
\end{aligned}
$$

2.4. The Validation of the Creep Model. Chen et al. [13] conducted uniaxial compression creep test to study the creep behavior of marble. The result showed that the long-term strength of marble is $130.1 \mathrm{MPa}$, and it underwent evident three-stage creep deformation when the axial compression stress exceeds 135.7 $\mathrm{MPa}$. Thus, the creep curve at 135.7 $\mathrm{MPa}$ is chosen to validate this creep model.

The calibrated parameters and simulated creep curves are shown in Table 1 and Figure 2, respectively. It reveals that the calculated results agree well with the test results, which shows that the creep model can describe the whole creep deformation stage of rock.

\section{Visco-elastoplastic Displacement of Tunnels}

3.1. Basic Assumption. The derivation of the visco-elastoplastic displacement of tunnels is based on the following assumptions (Figure 3):

(1) The radius of deep-buried tunnel is $R_{0}$. The hydrostatic pressure is $p_{0}$ and the tunnel is in the plane strain state.

(2) The creep deformation of surrounding rock occurs after the tunnel excavation. At this time, the stress redistribution of surrounding rock has been completed, and the secondary stress field of the surrounding rock is constant.

3.2. The Initial Stress Field before Creep Deformation of Tunnels. The surrounding rock of deep tunnels has nonlinear characteristics. Therefore, the Hoek-Brown criterion is suitable to describe the nonlinearity of rock mass. Its constitutive formula is

$$
\sigma_{1}=\sigma_{3}+\sqrt{m \sigma_{3} \sigma_{c}+s \sigma_{c}^{2}},
$$

where $\sigma_{1}$ and $\sigma_{3}$ are the maximum and minimum principal stress after the rock mass enters into the yield failure ( $\mathrm{MPa})$, $\sigma_{c}$ is the uniaxial compression strength of intact rock $(\mathrm{MPa})$,
TABLE 1: Creep parameters of marble.

\begin{tabular}{lcccc}
\hline$E_{1}(\mathrm{GPa})$ & $\eta^{\gamma}\left(\mathrm{GPa} \cdot h^{\gamma}\right)$ & $\eta 3(\mathrm{GPa} \cdot h)$ & $\gamma$ & $n$ \\
\hline 43.6 & 2465 & 3543 & 0.4 & 1.5 \\
\hline
\end{tabular}

and $m$ and $s$ are parameters related to the quality of rock mass. [10]

The stress in the elastic zone after tunnel excavation is

$$
\left\{\begin{array}{l}
\sigma_{r}^{e}=p_{0}-\frac{R_{0}^{2}}{r^{2}} N e^{2 M}, \\
\sigma_{\theta}^{e}=p_{0}+\frac{R_{0}^{2}}{r^{2}} N e^{2 M}, \\
\sigma_{z}^{e}=p_{0}, \\
M=\frac{\sqrt{m^{2}+16 m p_{0} / \sigma_{c}+16 s}-4 \sqrt{s}-m}{2 m} \\
N=p_{0}-\sqrt{s} \sigma_{c} M-\frac{m \sigma_{c}}{4} M^{2} .
\end{array}\right.
$$

The stress in the plastic zone is [10]

$$
\left\{\begin{array}{l}
\sigma_{r}^{p}=\frac{m \sigma_{c}}{4}\left(\operatorname{In} \frac{r}{R_{0}}\right)^{2}+\sqrt{s} \sigma_{c} \operatorname{In} \frac{r}{R_{0}} \\
\sigma_{\theta}^{p}=\frac{m \sigma_{c}}{4}\left(\operatorname{In} \frac{r}{R_{0}}\right)^{2}+\left(\sqrt{s} \sigma_{c}+\frac{m \sigma_{c}}{2}\right) \operatorname{In} \frac{r}{R_{0}}+\sqrt{s} \sigma_{c} \\
\sigma_{z}^{p}=\frac{(1+\sin \psi) \sigma_{\theta}^{p}+(1-\sin \psi) \sigma_{r}^{p}}{2} \\
R_{p}=R_{0} e^{M}
\end{array}\right.
$$

where $\sigma_{r}^{e}, \sigma_{\theta}^{e}$, and $\sigma_{z}^{e}$ are the radial, tangential, and normal elastic stress, respectively, $\sigma_{r}^{p}, \sigma_{\theta}^{p}$, and $\sigma_{z}^{p}$ are the radial, tangential, and normal plastic stress, respectively, $R_{p}$ is the radius of plastic zone, $r$ is the distance between any point within the surrounding rock and the circle center, and $\psi$ is the dilation angle.

\subsection{Creep Deformation}

3.3.1. Viscoelastic Zone. Figure 1 shows that the NVPB plastic element does not contribute to the creep deformation of tunnels when the surrounding rock is at the viscoelastic state. Thus, the creep model degrades into a creep model in which the elastic element connects with the Abel dashpot. The creep feature of surrounding rock at the elastic zone is described using this degraded model. 


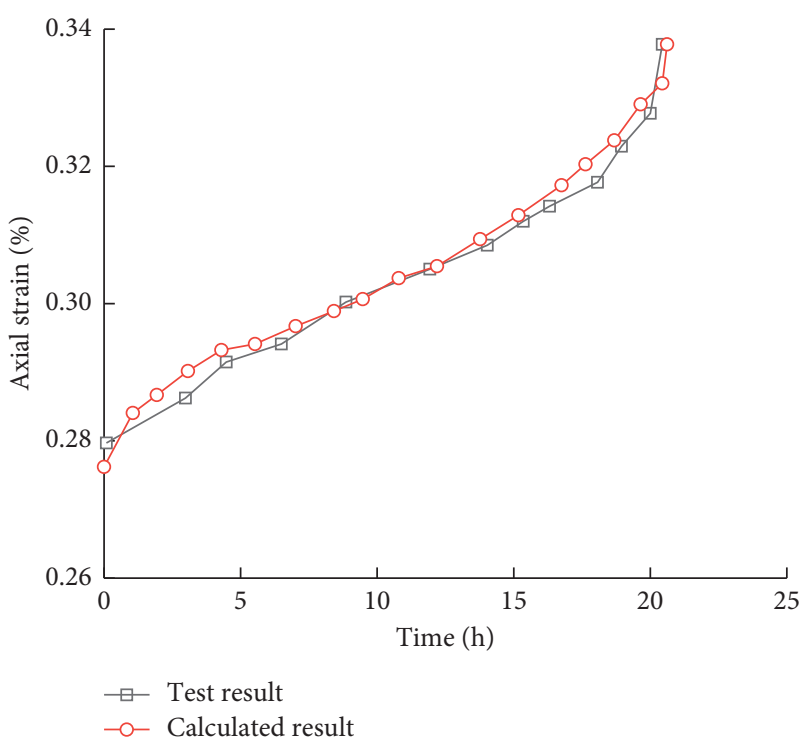

FIgURE 2: Validation of the creep model.

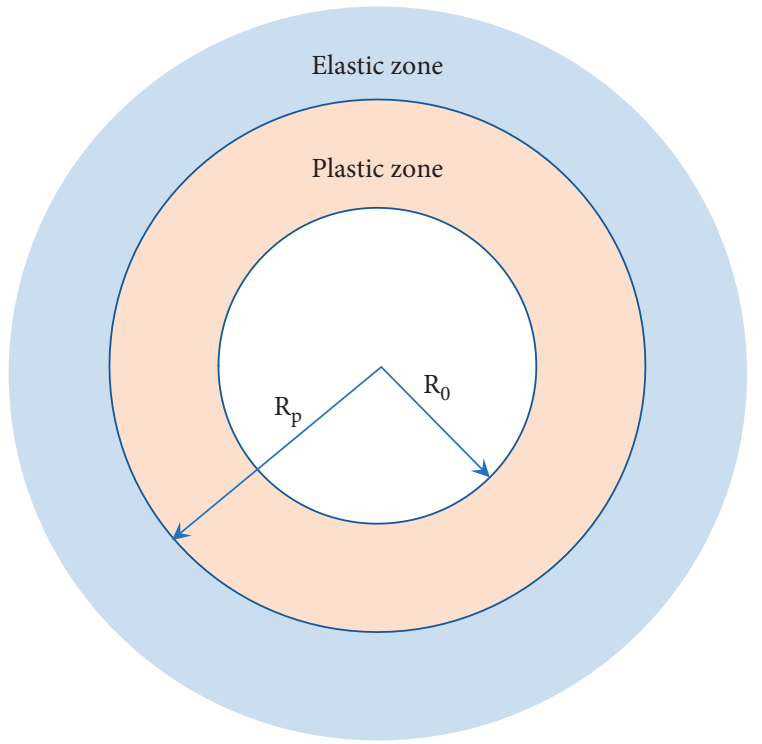

FIgure 3: Calculation model.

The relationship between deformation and strain of surrounding rock at the viscoelastic zone is

$$
\left\{\begin{array}{l}
\varepsilon_{\theta}=\frac{u}{r}, \\
\varepsilon_{r}=\frac{\mathrm{d} u}{\mathrm{~d} r},
\end{array}\right.
$$

where $\varepsilon_{\theta}$ and $\varepsilon_{r}$ are the tangential and radial strain of surrounding rock and $u$ is the radial deformation of surrounding rock.

Thus, the creep deformation is

$$
u_{r}^{\eta e}=\frac{R_{0}^{2}}{2 E r} N e^{2 M}+\frac{R_{0}^{2}}{r} N e^{2 M} \frac{t^{\gamma}}{\eta^{\gamma} \Gamma(1+\gamma)} .
$$

3.3.2. Viscoplastic Zone. The nonassociated rule is adopted to calculate the deformation at the viscoplastic zone:

$$
\frac{\mathrm{d} u_{r}^{\eta p}}{\mathrm{~d} r}+k_{\psi} \frac{u_{r}^{\eta p}}{r}=\varepsilon_{r}^{\eta p}+k_{\psi} \varepsilon_{\theta}^{\eta p},
$$

where $u_{r}^{\eta e}$ is the radial displacement at the viscoplastic zone and $k_{\psi}$ is the dilated coefficient:

$$
k_{\psi}=\frac{\tan (\pi / 4+\varphi / 2)}{\tan (\pi / 4+\varphi / 2-\psi)},
$$

where $\varphi$ is the frictional angle of rock mass.

Substituting equation (15) into equation (18) yields

$$
\begin{aligned}
\frac{\mathrm{d} u_{r}^{\eta p}}{\mathrm{~d} r}+k_{\psi} \frac{u_{r}^{\eta p}}{r}= & \frac{1}{6}\left[\frac{1}{2 E}+\frac{1}{\eta^{\gamma}} \frac{t^{\gamma}}{\Gamma(1+\gamma)}+\frac{t^{n}}{\eta_{3}}\right], \\
& \cdot\left[\left(k_{\psi}(3-\sin \varphi)-(3+\sin \varphi)\right)\right. \\
& \left.\cdot\left(\frac{m \sigma_{c}}{2} \operatorname{In} \frac{r}{R_{0}}+\sqrt{s} \sigma_{c}\right)\right], \\
& -\left(1+k_{\psi}\right) \frac{\sigma_{s} t^{n}}{\eta_{3}} .
\end{aligned}
$$

The displacement at the boundary between the viscoelastic and viscoplastic zone meets the following relation:

$$
\begin{aligned}
u_{r}^{\eta p}\left(R_{p}, t\right) & =u_{r}^{\eta e}\left(R_{p}, t\right), \\
& =\frac{R_{0}^{2}}{2 \mathrm{ER}_{p}} N e^{2 M}+\frac{R_{0}^{2}}{R_{p}} N e^{2 M} \frac{t^{\gamma}}{\eta^{\gamma} \Gamma(1+\gamma)} .
\end{aligned}
$$

Thus, the displacement at the viscoplastic zone, $u_{r}^{\eta p}$, is

$$
\begin{aligned}
u_{r}^{\eta p}= & \frac{1}{6}\left[\frac{m \sigma_{c}}{2\left(k_{\psi}+1\right)} g_{1}(r)+\left(r-R_{p}\left(\frac{R_{p}}{r}\right)^{k_{\psi}}\right) \sqrt{s} \sigma_{c}\right] \\
& \cdot\left[k_{\psi}(3-\sin \psi)-(3+\sin \psi)\right] \\
& \cdot\left[\frac{1}{2 E}+\frac{1}{\eta^{\gamma}} \frac{t^{\gamma}}{\Gamma(1+\gamma)}+\frac{t^{n}}{\eta_{3}}\right]+\left(r-R_{p}\left(\frac{R_{p}}{r}\right)^{k_{\psi}}\right) \frac{\sigma_{0} t^{n}}{\eta_{3}} \\
& +\left(\frac{R_{p}}{r}\right)^{k_{\psi}-1}\left[\frac{R_{0}^{2}}{2 \mathrm{ER}_{p}} N e^{2 M}+\frac{R_{0}^{2}}{R_{p}} N e^{2 M} \frac{t^{\gamma}}{\eta^{\gamma} \Gamma(1+\gamma)}\right],
\end{aligned}
$$

where $g_{1}(r)$ is

$$
\begin{aligned}
g_{1}(r)= & \operatorname{In}\left(\frac{r}{R_{0}}\right)^{r}-\left(\frac{R_{p}}{r}\right)^{k_{\psi}} \operatorname{In}\left(\frac{R_{p}}{R_{0}}\right)^{R_{p}} \\
& -\frac{1}{k_{\psi}+1}\left(r-R_{p}\left(\frac{R_{p}}{r}\right)^{k_{\psi}}\right) .
\end{aligned}
$$




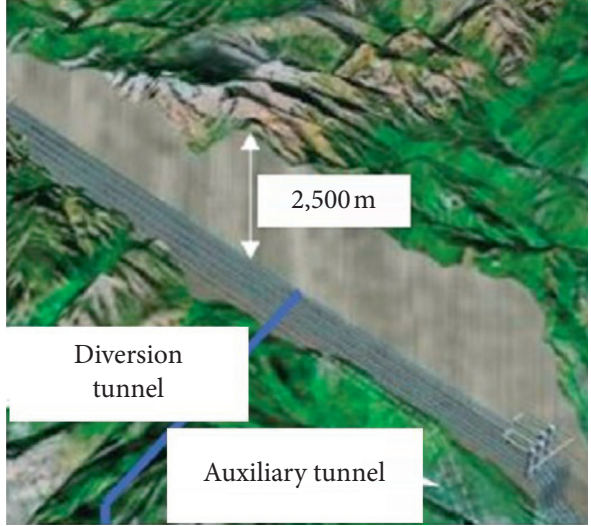

(a)

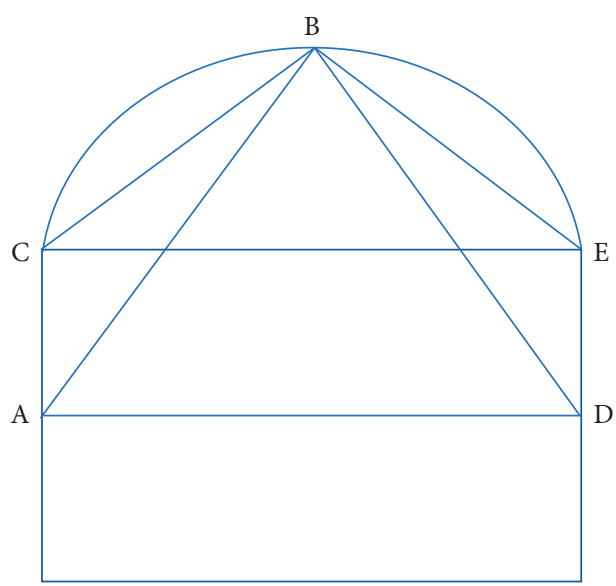

(b)

FIGURE 4: Jinping II hydropower station. (a) The position of tunnels [13]. (b) Measuring lines of the auxiliary tunnel [14].

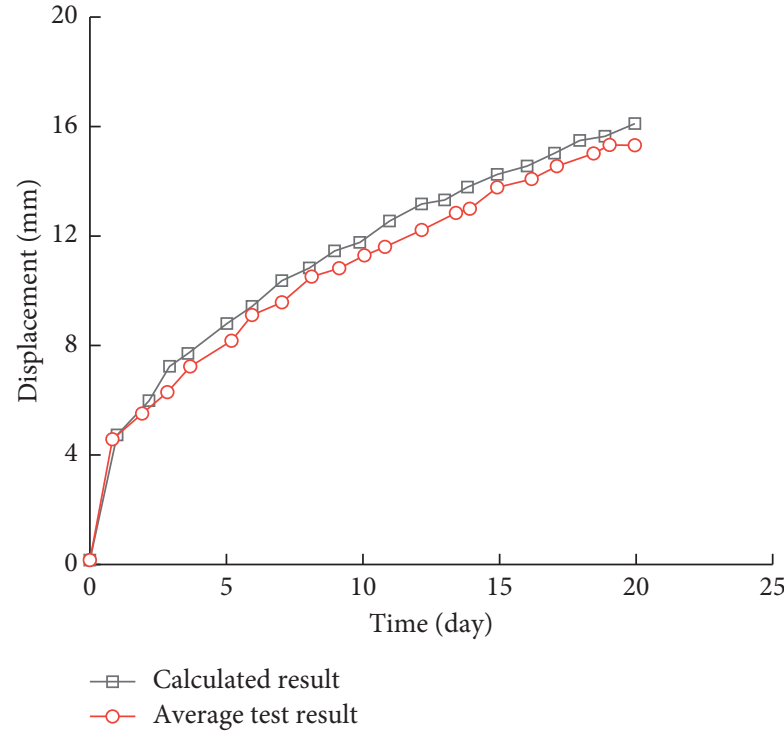

FIGURE 5: Comparison between analytical and test curves.

\section{Engineering Application}

4.1. Validation of the Closed-Form Solution. The Jinping number II hydropower station was built on the Jinping Dahe Bay in Sichuan Province (Figure 4(a)). This project consists of 4 diversion tunnels, 2 auxiliary tunnels, and 1 construction drainage tunnel. The average length of the tunnel is about $16.8 \mathrm{~km}$, and the average buried depth is between 1500 and $2000 \mathrm{~m}$.

The stratum that the auxiliary tunnel passes through is mainly composed of marble, and its uniaxial compressive strength is $141.17 \mathrm{MPa}, \mathrm{GSI}=50$, and $\mathrm{mi}=9$. According to the test results, the rock rheological parameters obtained by inversion are shown in Table 1. The initial in situ stress field of the tunnel site is simplified to the hydrostatic pressure field, that is, $p_{0}=40 \mathrm{MPa}$. The arrangement of the measurement points of the convergence monitoring section is shown in Figure 4(b).

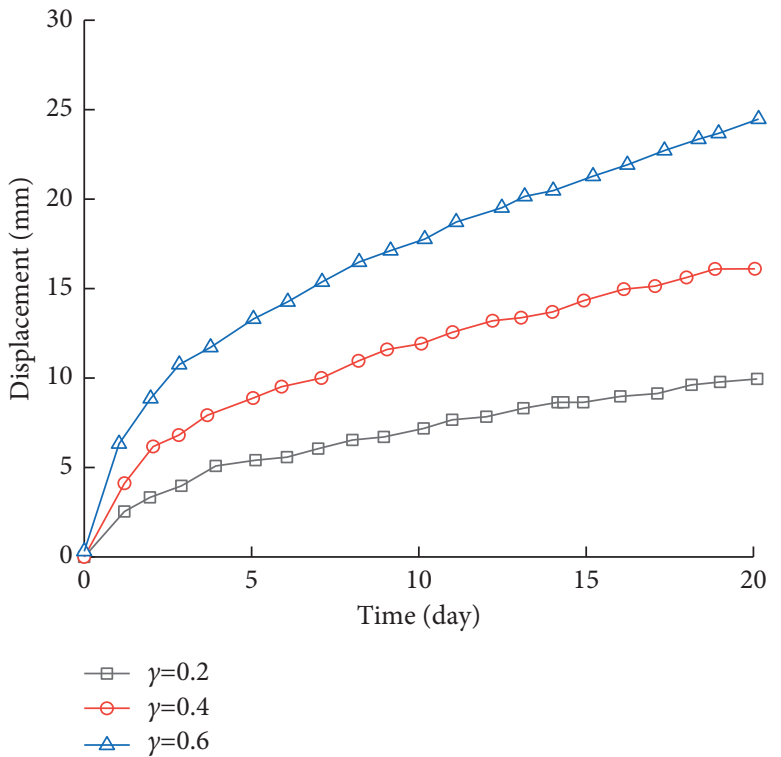

Figure 6: Influence of fractional order of Abel dashpot on the deformation.

The displacement curves of field monitoring and theoretical calculation is shown in Figure 5. It can be seen that the average value of the monitoring curve is in good agreement with the theoretical calculation result, which verifies the validity of the theoretical solution in this paper.

\subsection{Parametric Study}

4.2.1. The Influence of Fractional Order. The relationship between the order $\gamma$ of the Abel dashpot and the displacement of surrounding rock is shown in Figure 6. It shows that there is a positive correlation between the displacement of the surrounding rock and the magnitude of $\gamma$. This is because that, as the value of $\gamma$ increases, the viscous characteristic of the Abel dashpot becomes more and more 


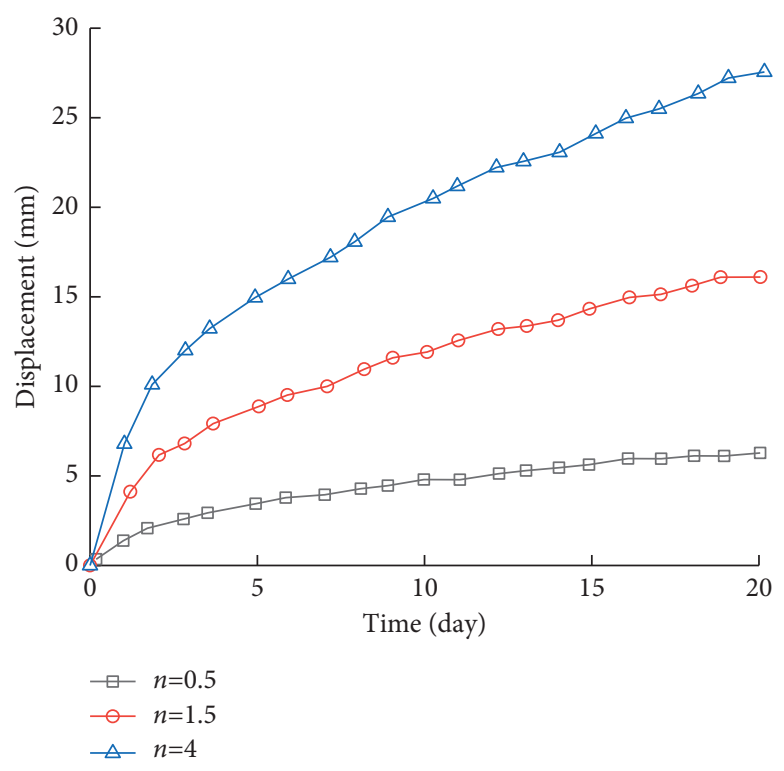

Figure 7: Influence of the creep index of NVPB element on the deformation.

obvious, which leads to an increase in the creep displacement of the surrounding rock.

4.2.2. Influence of the Rheological Index of NVPB Element. The influence of the rheological index of NVPB element on surrounding rock displacement is shown in Figure 7. It reveals that the displacement of surrounding rock increases with the increase of the rheological index. This is because, with the increase of the rheological index, the accelerating rheological process of the surrounding rock becomes more obvious, leading to an increase in the creep displacement of surrounding rock.

\section{Conclusion}

A creep model combining the Abel dashpot and a nonNewton viscous element was adopted, and the analytical solution about the visco-elastoplastic deformation for circular tunnel was derived based on this creep model. Major conclusions are as follows:

(1) The creep model can well describe the whole creep stage of rocks, that is, the decay, constant, and accelerated creep stages.

(2) The creep deform has a positive relation with the value of fractional order of Abel dashpot and the order of the non-Newton viscous element.

(3) The creep curves between test results and analytical solutions are well consistent with each other, which demonstrates the validity of the analytical solution.

\section{Data Availability}

The data used to support the findings of this study are available from the corresponding author upon request.

\section{Conflicts of Interest}

The authors declare that they have no conflicts of interest regarding the publication of this paper.

\section{Acknowledgments}

The research was supported by the National Natural Science Foundation of China (Grant no. 41977226).

\section{References}

[1] G. Xu, C. He, Y. Wang, and Y. Wang, "Study on the safety performance of cracked secondary lining under action of rheological load," China Civil Engineering Journal, vol. 49, no. 12, pp. 114-123, 2016.

[2] G. Y. Hong, J. J. Li, B. Qiu, and J. J. Li, "Solving equation of rheological deformation in axisymmetric round well under dead load," Rock and Soil Mechanics, vol. 32, no. 2, pp. 341346, 2011.

[3] S. L. Tang, H. Wang, J. Yu, J. Zhang, and Y. Cai, “Time-varying displacement analytical solution of deep soft rock tunnel considering strain-hardening and dilatation of surrounding rock," Tunnel Construction, vol. 37, no. 1, pp. 72-78, 2017.

[4] Z. J. Zhang, J. Yu, Y. Y. Cai, S.-H. Chen, and C.-M Lin, "Viscoelastic-plastic creep solutions and deformation properties of tunnels in swelling rocks under seepage," Chinese Journal of Geotechnical Engineering, vol. 36, no. 12, pp. 2195-2202, 2014.

[5] X. B. Gu and L. Yan, "Visco-elasto-plastic creep solutions to circular tunnels considering seepage force," Electronic Journal of Geotechnical Engineering, vol. 18, pp. 2231-2241, 2013.

[6] F. Deleruyelle, T. A. Bui, H. Wong, N. Dufour, D. K. Tran, and X. S. Zhang, "Analytical study of the post-closure behaviour of a deep tunnel in a porous creeping rock mass," Comptes Rendus Mecanique, vol. 344, no. 9, pp. 649-660, 2016.

[7] H. Lin, X. Zhang, Y. Wang et al., "Improved nonlinear Nishihara shear creep model with variable parameters for rock-like materials," Advances in Civil Engineering, vol. 2020, pp. 1-15, 2020. 
[8] H. Lin, X. Zhang, R. Cao, and Z. Wen, "Improved nonlinear Burgers shear creep model based on the time-dependent shear strength for rock," Environmental Earth Sciences, vol. 79, no. 6 , p. 149,2020

[9] J.-Z. Zhang, X.-P. Zhou, and P. Yin, "Visco-plastic deformation analysis of rock tunnels based on fractional derivatives," Tunnelling and Underground Space Technology, vol. 85, pp. 209-219, 2019.

[10] Y. Cai, J. Z. Zhang, J. Yu, and S.-H. Chen, "Nonlinear displacement solutions for deep tunnels considering whole process of creep and dilatation of surrounding rock," Rock and Soil Mechanics, vol. 36, no. 7, pp. 1831-1839, 2015.

[11] A. A. Kilbas, H. M. Srivastava, and J. J. Trujillo, Theory and Applications of Fractional Differential Equations, Elsevier, Amsterdam, Netherlands, 2006.

[12] W. Y. Xu, S. Q. Yang, and W. J. Chu, "Nonlinear viscoelastoplastic rheological model (HOHAI MODEL) of rock and its engineering application," Chinese Journal of Rock Mechanics and Engineering, vol. 25, no. 3, pp. 433-447, 2006.

[13] B. R. Chen, X. J. Zhao, X. T. Feng, and H. Zhao, "Time-dependent damage constitutive model for the marble in the Jinping II hydropower station in China," Bulletin of Engineering Geology and the Environment, vol. 73, pp. 499-515, 2014.

[14] B. R. Chen and X. T. Feng, "Universal viscoelastoplatic combination model and its engineering application," Chinese Journal of Rock Mechanics and Rock Engineering, vol. 27, no. 5, pp. 1028-1035, 2008. 